\title{
Philosophiques
}

\section{Le cercle et le doublet : Note sur Sartre et Foucault}

\section{Philip Knee}

Volume 17, numéro 1, printemps 1990

URI : https://id.erudit.org/iderudit/027106ar

DOI : https://doi.org/10.7202/027106ar

Aller au sommaire du numéro

Éditeur(s)

Société de philosophie du Québec

ISSN

0316-2923 (imprimé)

1492-1391 (numérique)

Découvrir la revue

Citer cette note

Knee, P. (1990). Le cercle et le doublet : Note sur Sartre et Foucault. Philosophiques, 17(1), 113-126. https://doi.org/10.7202/027106ar

\section{Résumé de l'article}

Ce texte esquisse deux voies possibles pour un dialogue entre Sartre et Foucault : la lecture des ambivalences de la pensée sartrienne sur le sujet, la morale, la politique, à travers la caractérisation de l'épistémè moderne dans Les mots et les choses; et, inversement, l'approche critique de la conception foucaldienne du savoir et du pouvoir à partir de l'anthropologie de la Critique de la raison dialectique. d'utilisation que vous pouvez consulter en ligne.

https://apropos.erudit.org/fr/usagers/politique-dutilisation/ 
PHILOSOPHIQUES, Vol. XVII, Numéro 1, Printemps 1990

\title{
NOTE DE RECHERCHE
}

\author{
LE CERCLE ET LE DOUBLET : \\ NOTE SUR SARTRE ET FOUCAULT \\ par Philip Knee
}

\begin{abstract}
RÉSUMÉ. Ce texte esquisse deux voies possibles pour un dialogue entre Sartre et Foucault: la lecture des ambivalences de la pensée sartrienne sur le sujet, la morale, la politique, à travers la caractérisation de l'épistémè moderne dans Les mots et les choses; et, inversement, l'approche critique de la conception foucaldienne du savoir et du pouvoir à partir de l'anthropologie de la Critique de la raison dialectique.
\end{abstract}

ABSTRACT. This paper suggests two possible directions for a dialogue between Sartre and Foucault : a reading of the Sartrean ambivalences on the subject, morality and politics in the light of the characterization of the modern épistémè in The Order of Things; and, inversely, a critical approach of the Foucaldian conception of power and knowledge through the anthropology of the Critique of Dialectical Reason.

Nous voulons proposer quelques pistes pour réfléchir sur le rapport entre Sartre et Foucault, car cette mise en relation n'a pas que l'intérêt historique de mettre aux prises deux penseurs en apparence très différents et qui se sont attaqués durement ${ }^{1}$; elle recèle aussi quelques-uns des grands enjeux de l'éthique contemporaine et mérite à ce titre d'être menée avec soin. Ceci impliquerait toutefois un travail considérable de reprise des textes que nous

1. Voir en particulier, pour FoUCAULT, les entrevues dans $L a$ Quinzaine littéraire, $n^{\circ} S$, 1966, et $\mathrm{n}^{\circ} 46,1968$; et pour SARTRE, les entrevues dans La Quinzaine Littéraire, $\mathrm{n}^{\circ} 14$, 1966, et surtout dans L'Arc, $\mathrm{n}^{\circ} 30,1966$ (traduite dans Telos, $\mathrm{n}^{\circ}$ 9, 1971). M. POSTER esquisse une mise en perspective historique des deux trajets, dans son Foucault, Marxism and History, Polity Press, Cambridge, 1984, ch. I. 
n'engagerons pas ici. Nous nous limiterons dans cette note à mettre en évidence deux points qu'il conviendra de développer ailleurs : d'abord il nous paraît possible, par la fameuse caractérisation de l'épistémè moderne comme "analytique de la finitude » avec ses «doublets», dans Les mots et les choses 2, d'éclairer certaines difficultés bien connues de la pensée sartrienne ; ensuite, en inversant la lecture, d'envisager l'anthropologie dialectique de Sartre ${ }^{3}$ comme une alternative positive à la position de Foucault sur le pouvoir et le savoir, et de cette manière d'ouvrir peut-être quelques voies de dialogue.

On ne peut manquer d'être tenté de lire les grandes ambivalences de la pensée de Sartre, ses interrogations inachevées, à la lumière des doublets foucaldiens de l'empirique et du transcendantal, du cogito et de l'impensé ; d'autant plus que le noyau théorique à partir duquel se déploient les positions sartriennes, c'est d'abord sa définition particulière de la conscience comme sujet, ce sujet dont Foucault fait son principal adversaire philosophique. En inscrivant sa démarche dans la tradition du cogito cartésien et de la phénoménologie, L'être et le néant rejette, entre autres, l'inconscient freudien et le matérialisme marxiste ${ }^{4}$ au nom d'une réalité humaine identifiée au mouvement même de la conscience. Mais en même temps, dès la critique de l'Ego de Husserl et dès l'introduction de l'ontologie fondée sur l'intentionnalité et le préréflexif, Sartre n'en rejette pas moins tout Je substantiel : la conscience n'est que pure extériorité, dépassement d'elle-même, et la liberté souveraine s'identifie au néant. Loin d'être un «sujet fondateur», le sujet sartrien est éclaté en une multiplicité d'actes de conscience discontinus, et n'est identifiable qu'à travers les objets concrets du monde. Cette ambiguité de la conscience se trouve parfaitement

2. Les mots et les choses (MC), Gallimard, 1966, ch. IX. Voir aussi « Nietzsche, Freud, Marx », dans Nietzsche, Cahiers de Royaumont, Éd. de minuit, 1967.

3. Essentiellement dans Questions de métbode et Critique de la raison dialectique, Gallimard, 1960 ; puis dans L'idiot de la famille, Gallimard, 1971-72. Voir aussi les importants textes réunis dans Situations IX, Gallimard, 1972: «L'écrivain et sa langue », « L'anthropologie », et « Sartre par Sartre », sur son rapport au structuralisme.

4. L'être et le néant (EN), Gallimard, 1943, p. 88-93, et p. 669. 
concentrée dans la théorie de la mauvaise foi, où par son incapacité à réaliser, comme dit Sartre, une " coordination valable " de ses deux aspects de transcendance et de facticité 5 , la conscience est d'emblée minée par un soupçon qui l'habite. Cette conscience sans contenu ni centre est si fluide qu'elle n'existe qu'en cherchant sans cesse à se donner consistance par un mensonge à soi. Et l'on en connaît toutes les conséquences dans les rapports à autrui.

À partir de la guerre, Sartre va pour ainsi dire réaliser les données de cette ambivalence structurelle dans un immense débat avec le marxisme (en passant de la conscience à la praxis), puis de manière plus implicite avec la psychanalyse (en passant de la conscience au vécu) et avec les théories du langage; c'est-à-dire avec des sciences du sujet soupçonné, miné, éclaté. Mises en cause d'abord au nom de la conscience translucide, celles-ci sont ensuite constamment sollicitées pour rendre compte de l'enracinement de cette conscience, et pour donner corps à la liberté par la description de sa situation, de son aliénation, de sa reprise d'elle-même. Et même dans ses analyses les plus «structurales», quand Sartre étudie le langage de Flaubert dans les années soixante, l'ambivalence ne se dément pas, car la multiplicité des facettes du conditionnement se totalisent en un projet où la praxis demeure irréductiblement libre. On a souvent suggéré que, philosophiquement, MerleauPonty a mieux compris Marx et Freud et mieux pris en charge le décentrement en jeu dans les sciences humaines en délaissant toute philosophie de la conscience ${ }^{6}$; alors que la consciencialisme têtu de Sartre l'a fait osciller entre les adhésions, les rejets et les emprunts. Ce qui est sûr, c'est que même en tirant Marx et Freud du côté de l'autonomie de la conscience contre sa chosification, Sartre s'est posé lui-même en "maître du soupçon»; mais c'est d'un soupçon limité ou honteux, car alors même que le décentrement se manifeste en de vastes enquêtes objectives, l'horizon demeure toujours celui d'un retour du sujet à lui-même.

Sartre n'est pas le penseur du cogito; ni vraiment, comme on l'a aussi suggéré, celui de sa déchéance. Il illustrerait plutôt le

5. $E N$, p. 95.

6. L'excellent livre de B. SICHËRE, par exemple, montre bien en quoi Merleau-Ponty situe sa démarche « au bord » du marxisme et de la psychanalyse : Merleau-Ponty ou le corps de la philosophie, Grasset, 1982. 
redoublement du cogito et de l'impensé par lequel Foucault caractérise l'impasse des anthropologies contemporaines, car par son sujet problématisé Sartre se place dans un entre-deux philosophique où il ne cesse de rechercher à penser l'impensé, à rendre compte de l'Autre en soi. En ce sens, il est clairement mis en cause dans la critique par Foucault de la tentative de la phénoménologie ${ }^{7}$ de dépasser l'oscillation de l'épistémè moderne par son appel à l'expérience vécue : tentative vaine, puisque le doublet de l'homme comme objet et comme sujet de la connaissance est indépassable, et que la phénoménologie est irrémédiablement prise entre une pure conscience transcendantale (sans historicité) et une pure conscience historique (en quête de ses origines), et que ses essais de conciliation avec le marxisme, loin de dépasser cette impasse, en témoignent plutôt. ${ }^{8}$

On peut envisager dans le même sens la grande quête morale de Sartre, son "problème moral» toujours repris mais jamais articulé si ce n'est en un engagement qui est «toujours-déjàaction» avant toute théorisation possible - ce qui est bien le statut auquel renvoie le diagnostic de Foucault sur l'impossibilité

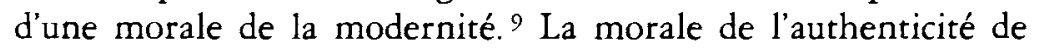
Sartre, mise en place mais non articulée dans L'être et le néant, procède du souci d'aménager moralement la «mort de Dieu », de donner sens à l'exigence morale dans le cadre d'un rejet de tout étalon. La valeur, tirant «son être de son exigence et non son exigence de son être " ${ }^{10}$, ne repose donc que sur le mouvement de la liberté créatrice. De ce fait, cette exigence sans fondement rationnel étant renvoyée à elle-même, le seul critère moral (défini par la théorie de la mauvaise foi) sera un critère formel de cohérence avec soi et de reprise de soi, appelé «authenticité » et «réflexion purifiante» ${ }^{11}$. On comprend dans ces conditions les difficultés de Sartre dans l'après-guerre. D'abord un humanisme embarrassé tente malgré tout d'asseoir des critères universels, puis la morale se cherche un contenu concret sur les terrains de la

\footnotetext{
7. Voir $M C$, p. 336-7 ; et le dialogue de conclusion de L'archéologie du savoir, Gallimard, 1969 , p. $259-275$.

8. $M C$, p. 332.

9. $M C$, p. 338-9.

10. $E N$, p. 76.

11. $E N$, p. 111 et p. 249,670 .
} 
totalisation historique et de la réciprocité biographique. Ces entreprises restent ancrées dans la morale de l'authenticité subjective tout en lui donnant une nouvelle signification: la praxis, dans Critique de la raison dialectique, retrouve tous les paradoxes de la mauvaise foi mais incarnés dans les «ensembles pratiques»d'où le statut éthique ambigu des catégories de rareté, de fraternitéterreur, de groupe-en-fusion. Dans L'Idiot de la famille, la fuite imaginaire, le qui perd gagne, l'aliénation à l'Autre, sont encore dénoncés, mais l'exigence qui motive cette dénonciation est liée à une autre : celle de comprendre par l'empathie et de rejoindre une universalité à travers les différences comprises. ${ }^{12}$

De cette manière, si la morale de Sartre reste un individualisme de la transparence, cette exigence ne vaut que portée par la compréhension de l'Autre et tendue vers le Nous; et c'est cette ambivalence qui est pratiquée et déployée par Sartre, mais jamais théorisée et dépassée. Si l'on peut parler d'un « nihilisme incomplet » dans L'être et le néant, où l'exigence de création des valeurs est renvoyée au formalisme de l'authenticité, le repli après L'être et le néant sur une morale historique et biographique reste lui-même incomplet et en quête de ses fondements. ${ }^{13}$ Sartre n'est donc pas le penseur du sujet moral, seulement celui de l'exigence morale, celui de la morale impossible depuis Kant. Car ce sujet n'existe que comme un perpétuel écart - un écart qu'il s'agit soit de montrer par un jeu d'alternatives dramatiques, soit de combler par une recherche effrénée de médiations, laquelle, faute d'une philosophie qui la fonde, ouvre sur un travail circulaire infini.

Corollairement, l'engagement politique de Sartre procède d'une recherche d'effectivité contre la tentation imaginaire dont il se sent prisonnier et contre une liberté qui reste séparée des autres et du temps historique - d'où ses combats contre les spectres de l'aventurier, du comédien, du marginal, traîtres en puissance dans le monde de l'action politique. ${ }^{14}$ Mais on sait que son adhésion au marxisme devient une critique constante du mécanisme au nom de

12. C'est la problématique de « l'universel singulier $\%$, qui commande l'étude sur Flaubert; L'idiot de la famille, préface.

13. Le farouche anti-humanisme des premières auvres littéraires de Sartre est modéré dans l'EN, puis pratiquement abandonné.

14. Voir Les Mains sales et Le Diable et le Bon Dieu, notamment. 
la praxis ; et cette critique s'appuie précisément sur la liberté que Sartre tentait de fuir, piégeant ainsi sa pensée politique et expliquant en partie les retournements et les rigidités de celle-ci. ${ }^{15}$ Dès les années cinquante, c'est encore Merleau-Ponty qui aperçoit les difficultés de la tentative de "trouver à la liberté une ligne politique » ${ }^{16}$; mais c'est dans les années soixante et soixante-dix que ces difficultés se révèlent le mieux, par l'affinité de Sartre avec des pratiques militantes (très proches de celles de Foucault - nous y reviendrons) qui combinent justement les exigences de la transformation sociale et celles de l'imaginaire; qui investissent le champ de l'action politique avec un modèle de pure négativité créatrice se refusant à toute médiation institutionnelle, et qui débouchent alors sur la violence au nom d'une démystification ou d'une résistance permanentes. ${ }^{17}$ Si Sartre se retrouve au plus près de ces pratiques, c'est que ses propres positions ne cessent d'osciller entre la critique acerbe de l'atomisme social, de tout libéralisme, au nom d'une utopie communautaire fusionnelle; et la critique de toute politique prétendant boucler l'existence collective en une totalité, au nom cette fois de la praxis individuelle. Sartre n'est donc pas le champion d'une politique moralisée, mais celui d'un activisme exprimant ces options contradictoires de manière exacerbée en les renvoyant l'une à l'autre selon les conjonctures; celui d'une contestation qui refuse de prendre en charge ces exigences pour les gérer dans des institutions où s'articuleraient l'affirmation et la nécessaire limitation de la liberté ouvrant ainsi aux acteurs sociaux un espace qui serait proprement politique, du moins dans le sens donné à ce terme à l'époque moderne. À son corps défendant peutêtre, dans la mesure où il se réclame le plus souvent du marxisme, Sartre est ainsi l'un des acteurs essentiels d'une mise en cause profonde de la pratique politique dans cette période - une mise en cause dont son œuvre porte témoignage sans doute, mais dont Foucault, surtout, a tenté de rendre compte théoriquement, particulièrement dans sa critique de la conception du droit et du pouvoir

15. Le récent essai d'E. BARILIER met bien en évidence les implications politiques d'une liberté conçue par Sartre sur le mode de la création artistique : Les petits camarades, L'Âge d'homme/Julliard, 1987.

16. Merlead-Ponty, Les aventures de la dialectique, Gallimard, 1955, p. 257

17. Voir, entre autres, les textes sur le colonialisme dans Situations $V$, Gallimard, 1964, et ceux sur Mai 68 dans Situations VIII, 1972, et Situations X, 1976. 
fondée sur l'idée de souveraineté et héritée des Lumières, dont il affirme la caducité. ${ }^{18}$

Mais on ne peut se limiter à une mise en perspective archéologique de Sartre par Foucault sans interroger le statut de son propre discours. Or il apparaît, comme l'ont noté par exemple Dreyfus et Rabinow, ${ }^{19}$ que la méthode foucaldienne reste ellemême "prisonnière du double", qu'elle est elle-même "une variante de la finitude ». En effet, on sait que Foucault se refuse à tout horizon de sens tel que préétabli par ceux dont il discute les pratiques discursives, afin d'expliciter la formation des ensembles discursifs selon des règles indépendantes de ce sens. Mais il ne peut se détacher totalement de cet horizon de sens sans que les discours étudiés ne sombrent dans l'indistinction. Il doit bien les comparer, ne serait-ce, par exemple, que pour établir sa périodisation des épistémès; et il doit donc le faire à partir d'un point de vue : son présent historique comme chercheur. ${ }^{20} \mathrm{Il}$ est donc contraint de se donner un certain détachement (au nom du caractère illusoire du sens établi par les sujets), et de se plonger dans cet univers de sens pour comprendre ces discours. D'où la reproduction d'un doublet par lequel Foucault esquive la question des fondements et du lieu de son propre discours ${ }^{21}$, en oscillant entre la connaissance détachée et la compréhension du sens. Nous mentionnons cette difficulté moins pour la discuter comme telle que parce qu'elle suggère que le problème du doublet n'est peut-être pas dépendant du caractère antbropologique des pensées qui en sont affectées (comme le serait celle de Sartre, selon Foucault) ; en fait, le doublet renvoie à une structure plus fondamentale de la pensée. Dès lors l'enjeu est moins de le repérer pour le dénoncer, quitte par là à en

18. Sur la conception du pouvoir, voir essentiellement La volonté de savoir, Gallimard, 1976, p. 121-135; et sur la critique du juridisme et des théories du contrat : Surveiller et Punir, Gallimard, 1975, surtout ch. II.

19. H. Dreyfus et P. Rabinow, Michel Foucault, un parcours philosophique, Gallimard, 1984, p. 145

20. C'est l'un des arguments de Habermas contre le « cryptonormativisme » de Foucault : Le discours philosophique de la modernité, Gallimard, 1988, p. 329-330.

21. L'archéologie du savoir, p. 267. 
être soi-même victime à son insu et sans possibilité de maîtrise, que de le prendre en charge et d'en explorer les facettes et les implications. Ne peut-on pas envisager sous cet angle les efforts de Sartre, comme une prise en charge de l'analytique de la finitude au sein de sa philosophie anthropologique, de manière à assumer en toute lucidité le problème de l'impensé et celui des doublets? Cette hypothèse nous semble d'autant plus légitime, qu'elle permet d'envisager en même temps l'autre grande difficulté de la pensée de Foucault concernant le statut de la vérité dans le cadre de sa critique du sujet et de l'autonomie du savoir. Renvoyant tout savoir aux conditions de son émergence, y compris le savoir qu'on peut avoir de ces conditions, faisant du pouvoir le « régime interne » du savoir, et de la vérité « l'effet» des différents régimes discursifs ${ }^{22}$, on voit sans peine la charge épistémologique à laquelle s'expose cette pensée, celle de sombrer dans le relativisme ou du moins dans un scepticisme qui met en question ses propres énoncés sur le pouvoir.

L'alternative sartrienne trouve son expression théorique la plus nette dans la Critique de la raison dialectique et ses descriptions de l'envers pratico-inerte de la praxis. ${ }^{23}$ Le pratico-inerte est ce niveau de socialité où une multiplicité de praxis se conditionnent par leur appartenance à un même champ de matérialité, et produisent ce que Sartre appelle des « contre-finalités ». Il y a donc une dimension d'inertie au cœur de la praxis, qui prend la forme d'exigences, d'impératifs, de normes s'imposant à chacun et définissant un futur anonyme comme devant-être-réalisé. Cette contrainte n'est pas le résultat d'une imposition extérieure sur les praxis, mais elle est immanente au champ social. De cette manière

22. Sur la critique d'inspiration nietzschéenne de la vérité: "Nietzsche, la généalogie, I'histoire ", dans Hommage à Jean Hyppolite, P.U.F., 1971, p. 149-150; et L'Ordre du discours, Gallimard, 1971, p. 15-20. Foucault écrit par ailleurs à ce sujet : « La vérité est de ce monde ; elle y est produite grâce à de multiples contraintes. Et elle y détient des effets réglés de pouvoir. Chaque société a son régime de vérité, sa "politique générale" de la vérité : c'est-à-dire les types de discours qu' elle accueille et fait fonctionner comme vrais ; les mécanismes et les instances qui permettent de distinguer les énoncés vrais ou faux, la manière dont on sanctionne les uns et les autres; les techniques et les procédures qui sont valorisées pour l'obtention de la vérité ; le statut de ceux qui ont la charge de dire ce qui fonctionne comme vrai » : «Vérité et pouvoir », dans $L^{\prime} A r c, n^{\circ} 70$, 1977 , p. 25.

23. Critique de la raison dialectique, surtout le Livre $\mathrm{I}$. 
Sartre peut rendre compte des conditionnements et des effets anonymes des praxis sans que celles-ci soient réduites à des mécanismes. L'autonomie pratique des agents comme libres praxis totalisatrices est maintenue, tout en explicitant la passivité qui les habite: l'Autre en eux qui menace toujours de leur dérober le sens de leurs actes. Sartre fait ainsi une description de la praxis comme un mouvement de totalisation du conditionnement et du dépassement de ce conditionnement, de son intériorisation et de sa réextériorisation. Cette description se fonde, non sur un recul réflexif qui réduirait l'objet à des principes explicatifs extérieurs, mais sur une vérité conçue comme une interaction constante de la connaissance de l'être et de l'être de la connaissance, où la pensée est à la fois constituée et constituante. D'où une dialectique circulaire, chez Sartre, comme totalisation de l'histoire et de la connaissance ${ }^{24}$, dont on aperçoit la double pertinence par rapport à Foucault.

D'abord, cette circularité dialectique peut être vue comme prenant en charge la "grande oscillation " ${ }^{25}$ des doublets, c'està-dire l'interrogation sur les conditions de possibilité de la connaissance qui porte justement sur un objet de la connaissance empirique : l'homme. De l'incessant jeu de renvois de l'analytique de la finitude, Sartre fait sa règle rigoureuse, en liant dans le mouvement de totalisation de la praxis et dans la circularité de la connaissance et de la compréhension de la méthode historique et biographique, les pôles de l'empirique et du transcendantal, du cogito et de l'impensé. Dès lors peut-on encore parler d'un doublet et de son inévitable échec, quand les deux aspects sont les moments d'un mouvement pratique qu'aucune théorie (ou dialectique) extérieure ne peut figer (la connaissance étant un moment de la praxis), et qui se poursuit dans l'inachèvement comme la condition même de son intelligibilité?

Ensuite, il est clair que la contre-finalité de Sartre est à rapprocher de la description par Foucault des effets anonymes d'un ensemble de pratiques ( intentionnelles » mais non articulées

24. Critique de la raison dialectique, introduction; er Questions de méthode, ch. III, sur la méthode "progressive-régressive » ou «analytique-synthétique », et la «connaissance compréhensive» (p. 111). C'est toute la démarche "critique» (au sens kantien) de Sartre qu'il faudrait examiner ici, à la lumière des analyses sur Kant dans $M C$.

25. $M C$, p. 383. 
et « non subjectives », dit-il ${ }^{26}$ ) qui produisent des effets de pouvoir échappant radicalement aux acteurs historiques. Plus précisément, certains textes de Foucault définissent la relation de pouvoir comme une «action sur l'action, sur des actions éventuelles ou actuelles, furures ou présentes », ce qui requiert que celui sur lequel elle s'exerce « soit bien reconnu et maintenu jusqu'au bout comme sujet d'action"; l'exercice du pouvoir, le "gouvernement» (au sens le plus large, dit Foucault), consistant ainsi à «conduire des conduites ". ${ }^{27}$ Mais Foucault ne théorise pas ce sujet dont il affirme ici l'incontournabilité, fût-ce comme «sujet perdu ». Ceci lui aurait permis, nous semble-t-il, de fonder philosophiquement la résistance au pouvoir, et d'éviter que celle-ci, définie comme intérieure aux relations de pouvoir, ne reste finalement indistincte de ces relations. Chez Sartre, en revanche, on sait que même quand la praxis remplace le pour-soi dans le développement de son œuvre et s'explicite en termes d'aliénation sociale, le sujet ne se réduit pas à des structures linguistiques ou sociales, Sartre n'abandonnant jamais totalement ses fondements ontologiques initiaux. Le champ social reste alors intelligible, non par le privilège donné jadis à la conscience, mais par la circularité praxis-processus évoquée plus haut, en ce que celle-ci explicite, par son va et vient, comment se constitue la contre-finalité dans les activités pratiques, comment se disperse et se perd l'autocompréhension des agents, mais sans que le chercheur se retrouve démuni devant des stratégies sans projet et des effets de pouvoir anonymes dont l'intelligibilité ne pourrait que lui échapper. Sartre affirmerait contre Foucault que le pouvoir ne gouverne les activités pratiques que dans la mesure où ce pouvoir est intériorisé comme exigence et par là même réextériorisé et transformé par une libre praxis ; en d'autres termes qu'il n'y a pas de détermination pratico-inerte sans une reprise totalisatrice. On voit alors les conséquences pour l'activité connaissante et l'écueil du relativisme évoqué plus haut pour Foucault : le savoir rationnel maintient une mesure d'autonomie irréductible chez Sartre, car il se donne ses propres règles en

26. La volonté de savoir, p. 124-5.

27. Osons avancer que Foucault se révèle singulièrement «sartrien » dans ce texte tardif sur le pouvoir, écrit pendant qu'il étudie la question du sujet pour les tomes 2 et 3 de l'Histoire de la sexualité : "Le pouvoir, comment s'exerce-t-il?", dans Dreyfus et RabinOw, op. cit., p. 313-4. 
réextériorisant le réseau de pouvoirs qui le conditionne, en même temps qu'il intériorise comme exigence pratico-inerte les impératifs de sa situation. Par la circularité de la connaissance et de l'être, de l'absolu et du relatif, Sartre cherche ainsi à maintenir une transhistoricité de la raison pratique, qui évite que la valeur ne s'effondre simplement en fait, ou le savoir en effet de pouvoir.

On sait bien sûr que Foucault rejette explicitement l'idée de totalisation ${ }^{28}$ et situe son entreprise en un tout autre registre que celle de la Critique de Sartre où on a encore affaire à une liberté se heurtant au pouvoir, et non à une déconstruction de réseaux et de stratégies débarrassée de toute tentation « finaliste ». Il n'empêche que si l'on consent à prendre au sérieux l'épistémologie de la praxis de cette dernière cuvre (ainsi que sa mise en pratique dans L'Idiot de la famille) et à y voir, plutôt qu'un anthropologisme dépassé, le prolongement et l'approfondissement théorique de ce sujet éclaté, discontinu et miné par son Autre, mis en place au début de l'œuvre sartrienne, on apercevrait peut-être des ressources capables de répondre à certaines des difficultés les plus incontournables de la pensée de Foucault concernant sa réduction du savoir au pouvoir et sa relativisation de la vérité.

Si ce dialogue peut être fructueux, il convient de ne pas l'envisager en termes de possibles convergences mais seulement d'échos entre ces deux pensées - des échos dont la résonance est toutefois considérable pour le problème éthique aujourd'hui, nous semble-t-il, comme peut le suggérer le lien entre deux points déjà aperçus et qui ne sont éloignés qu'en apparence: la référence à Nietzsche et l'activité politique dans les années soixante-dix en France. Il est significatif, en effet, que Sartre et Foucault, si différents sur tant de fronts, se soient retrouvés à partir de Mai 68 (et malgré les invectives des années précédentes) dans ce que J. Colombel appelle, pour y applaudir, une "philosophie de la

28. L'archéologie du savoir, p. 24. 
subversion » contre tous les pouvoirs. ${ }^{29}$ Leurs interventions et prises de position montrent qu'ils s'intéressent avant tout à la multiplication des luttes immédiates contre toutes les formes d'assujetissement, aux combats et aux résistances ponctuels et toujours renouvelés qui minent les pouvoirs en place et ne durent jamais assez pour se pétrifier en institutions (Sartre) ou basculer dans la normalisation (Foucault) - l'enjeu, loin d'être la prise du pouvoir, étant d'imaginer toujours mieux comment ne pas le prendre, par un incessant jeu de provocations et d'esquives. Cette période, en effet, n'est pas animée en profondeur par une revendication morale ou politique, mais plutôt par la mise en cause de tout ordre moral et politique: l'affirmation de l'individualité comme règne du relativisme des valeurs et de la «différence ». La signification philosophique de ce règne n'est évidemment pas l'humanisme du sujet mais son ébranlement, ce qui donne à Mai 68 sa force de symbole pour toute notre époque.

On peut comprendre alors que Foucault s'y soit retrouvé à son aise, car sa pensée se fonde sur le diagnostic nietzschéen de la maladie du monde moderne, sur un nihilisme qu'il veut assumer dans toutes ses implications en annonçant la mort de l'homme à partir de celle de Dieu et en construisant une philosophie déconstructrice de la volonté de vérité. Dans ce cadre, la présence militante de Sartre à ses côtés est suggestive, car contre les étiquettes trop univoques et contre Sartre lui-même, elle invite à envisager sa pensée à partir de ce même nihilisme, mais avec cette différence qu'il n'est justement jamais pris en charge comme tel par Sartre. Pour Foucault, il y aurait chez Sartre un nietzschéisme honteux, ou un humanisme désespéré, qui réagirait à la mort de Dieu en érigeant l'homme en absolu (ce qui est déjà en évidence dès le projer d'être Dieu, c'est-à-dire en soi - pour soi, dans L'être et le néant), Sartre étant incapable de lire jusqu'au bout le sens du

29. J. Colombel, Sartre, Textes et débats, Union générale d'édition, 1985, vol. 1, p. 397. Cetre commentatrice exagère les convergences entre Foucault et Sartre, nous semble t-il, en jugeant que ce dernier * aurait dû être sensible » à la méthode généalogique de Foucault de par son propre échec à rendre compte d'une « histoire globale ». Sans doute Sartre donne un " rôle positif aux ruptures et à la discontinuité » dans l'histoire, mais il nous semble néanmoins bien éloigné de "l'histoire générale » de L'archéologie du savoir, et plutôt du côté de cette " histoire des philosophes » que Foucault reconnaît vouloir a tuer " (Entrevue dans La Quinzaine littéraire, 1968, op. cit.). 
message nietzschéen. C'est, en revanche, d'avoir pris la mesure de cet événement et d'avoir fait le saut pour consentir à penser «dans le vide de l'homme disparu» ${ }^{30}$, qui permettrait à Foucault de se rire des charges de relativisme qu'on lui oppose. Mais cela ne rendil pas inaccessible à Foucault tout action morale et politique cohérente dans le présent, s'étant interdit toute justification philosophique de la résistance au nom de cet «à-venir 》 nietzschéen? Chez Sartre le diagnostic nihiliste est présent au départ, comme critique radicale de toute morale qui cherche à fuir la contingence, mais c'est comme si ce diagnostic ne restait à l'cuvre ensuite que sous la forme de la dénégation: toujours présent mais jamais discuté, jamais perdu de vue mais refusé dans ses conséquences, car Sartre veut maintenir à tout prix la possibilité d'une morale et d'une politique fondées en raison. C'est ce qui éloigne Sartre petit à petit de toute référence à Nietzsche, au profit des deux autres "maîtres du soupçon», Marx et Freud, qui sont loin d'avoir accompli la même rupture avec l'humanisme et la tradition des Lumières, et qui sont tout au plus des points d'appui, considérables mais vite dépassés, chez Foucault. D'où cette philosophie sartrienne si ambivalente et qui semble si facilement critiquable dès que, comme chez Foucault, tout ordre de vérité est ramené au pouvoir : une philosophie de la subjectivité qui mine ses propres conditions de possibilité mais ne lâche pas prise sur sa référence essentielle; une exigence morale qui s'interdit les moyens de son effectuation mais ne se renie pas; une quête de médiations politiques qui ne cesse d'échouer en retrouvant toujours ses propres fondements imaginaires, mais sans abandonner le terrain politique.

En définitive, entre les illusions d'une naïve philosophie du sujet et l'impasse d'un anti-humanisme qui prétend se détourner de l'éthique, l'interrogation contemporaine sur cette dernière semble bien souvent se ramener à cetre question brutale : que faire de Nietzsche aujourd'hui? Après lui, en d'autres termes, s'agit-il encore de chercher à penser l'impensé dans une perspective " moderne » pour laquelle la réflexion sur l'homme peut le changer, auquel cas la circularité dialectique sartrienne s'offre comme une méthode positive et la voie d'une éthique possible? Ou doit-on

30. $M C$, p. 353. A ce sujet, il est piquant de voir Foucault évoquer l'intérêt pour Nietzsche du jeune Sartre de 1925, et suggérer par là que leurs trajectoires respectives sont inverses: Entrevue dans Telos, no 55,1983 , p. 204. 
considérer que ce cercle ne masque qu'un doublet stérile et une nouvelle tentative pour saisir l'impensé en s'enfermant encore dans une modernité qui s'épuise à éprouver la finitude de l'homme? Par-delà les réputations trop rigides, il nous semble que c'est d'abord dans cette direction qu'il serait fécond de faire s'interroger l'un par l'autre Sartre et Foucault. *

Faculté de philosophie

Université Laval

* Ce texte reprend certains éléments d'une communication présentée au $3^{\mathbf{e}}$ colloque de la Sociéré Sartre d'Amérique du nord, à l'Université Duquesne, Pittsburgh, 30 sept. 2 oct. 1988 